\title{
A Study on the Dental Care Characteristics of Tetra-Tip Filament Toothbrush Bristle
}

\author{
ByungJae Park ${ }^{1}$, JaeBum Ahn ${ }^{1}$, Jongjin Ahn ${ }^{1}$ \\ ${ }^{1}$ BBC Co., Ltd., Daejeon, Korea (Republic of)
}

\begin{abstract}
Objectives: The tetra-tip filament bristle is a branched toothbrush bristle with four fine tips in a single filament body to improve shortcomings and enhance properties of conventional non-tapered or tapered filaments. The tetra-tip bristles showed better the capabilities of cleaning performance in brushing teeth compared to the non-tapered and tapered single filament.

Methods: The Tetra-tip filaments for bristles were manufactured by melt-spinning polymer through a spinneret comprising an orifice of tetra-core/sheath configuration, and then were branched out into fours tips with a diameter of approximately $0.18 \mathrm{~mm}$ and tip length within the range of $1.5-2.5 \mathrm{~mm}$ at the ends of them using chemical etching so called alkaline-hydrolysis. To evaluate the characteristics, non-tapered and single-tip tapered bristles were selected as controls. The structure and appearance of the bristles were analyzed through microscope, and tensile strength, bend recovery, and stiffness test were evaluated for the characteristics of them, and also repeated brushing - simulated frictional deformation tests were performed for durability evaluation. To evaluate the cleaning ability, plaque removal test was conducted in simulated brushing environment.

Results: It was confirmed that tapered four tips in a single body were formed stably through chemical etching. The tensile strength and bend recovery of the four-tips bristles was more than $400 \mathrm{~N} / \mathrm{mm}^{2}$ and more than $60 \%$, respectively. Also, it was founded that the stiffness value of tetra-tip was $0.68 \mathrm{~N}$ while single tip (tapered) and non-tapered bristle was $1.05 \mathrm{~N}$ and $1.87 \mathrm{~N}$, measured at $1 \mathrm{~mm}$ point away from tip end, respectively. In the durability test, there was no deformation under 50,000 cycle brushing conditions. The cleaning efficacy of tetra-tip in plaque removal test was all excellent compared to the control group.

Conclusions: The tetra-tip bristle was distinct in appearance, and showed good performance in terms of fiber strength, bend recovery, durability, resistance to deflection and flexibility, but the cleaning power was superior to other bristles. This result was believed to be due to reduced tooth irritation and improved contact area and micro penetration on teeth by fine branching tips. Therefore multi-tip bristles are not only suitable for users with poor oral conditions, but also have the advantage of providing customized solutions for user oral conditions through specification control.
\end{abstract}

Keywords: Toothbrush bristle, tetra-tip filament, multi-tip filament, melt spinning, filaments

Copyright (C) 2021. Korean Academy of Preventive Dentistry. All rights reserved.

This is an Open Access article distributed under the terms of the Creative Commons Attribution Non-Commercial License (http://creativecommons.org/licenses/ by-nc/4.0) which permits unrestricted non-commercial use, distribution, and reproduction in any medium, provided the original work is properly cited. 